\title{
The Influence of Parameters of Stabilized Corona Discharge on its Microbicidal Effect
}

\author{
V. Scholtz ${ }^{a, *}$, L. Kommováa And J. JulÁK ${ }^{b}$ \\ ${ }^{a}$ Department of Physics and Measurements, Faculty of Chemical Engineering, Institute of Chemical Technology \\ in Prague, Technická 5, 166 28, Praha, Czech Republic \\ ${ }^{b}$ Institute of Immunology and Microbiology, 1st Faculty of Medicine, Charles University in Prague \\ Studničkova 7, 128 00, Praha, Czech Republic \\ (Received July 16, 2010; in final form January 31, 2011)
}

\begin{abstract}
This work presents the study of microbicidal effects of positive flashing corona, negative pulseless corona and glow corona discharges and the comparison of their efficiency in the point-to-plane arrangement in water suspension. The flashing corona seems to be the most effective one, however the interval of optimal parameters is wide. The total inhibition of microorganisms becomes after $75 \mathrm{~s}$ or $8-10 \mathrm{~min}$ of exposition for bacteria or yeast, respectively. A hypothesis explaining the different sensitivity of various microbes is postulated.
\end{abstract}

PACS: 52.77.-j, 52.27.Lw

\section{Introduction}

Microbicidal effects of low-temperature plasma generated in electrical discharges are well established area of research and are the mainstream of study of several laboratories in the world. Despite the plenty of works handled with this problematic, the method is not yet frequently used in practice. Nevertheless, it is potentially important, especially for the decontamination or sterilization of heat labile or otherwise sensitive materials. Its various experimental arrangements, advantages and status of research in this field were reviewed in detail in many works, e.g., [1-4]. This study is focused on the application of corona discharge for the decontamination of water suspension of microorganisms. We attempted to compare the microbicidal efficiency and energetic content of various types of discharges of both polarities.

\section{Used apparatus}

The low temperature plasma was generated using the previously described $[5,6]$ simple apparatus of an open-air type. Briefly, the negative or positive corona discharges were generated on the point electrode represented by the tip of a syringe needle, stabilized by the serial resistance of $20 \mathrm{M} \Omega$ connected between the source of $\mathrm{DC}$ high voltage and the plane electrode, realized by the surface of water suspension of microorganisms grounded

\footnotetext{
* corresponding author; e-mail: scholtzv@vscht.cz
}

with an immersed platinum wire. A micrometer screw adjusted the position of the coronizing electrode. The used source HT 2103 (Utes Brno, Czech Republic) made it possible to set a variable voltage up to $10 \mathrm{kV}$ and current up to $0.5 \mathrm{~mA}$. The experimental arrangement is depicted schematically in Fig. 1.

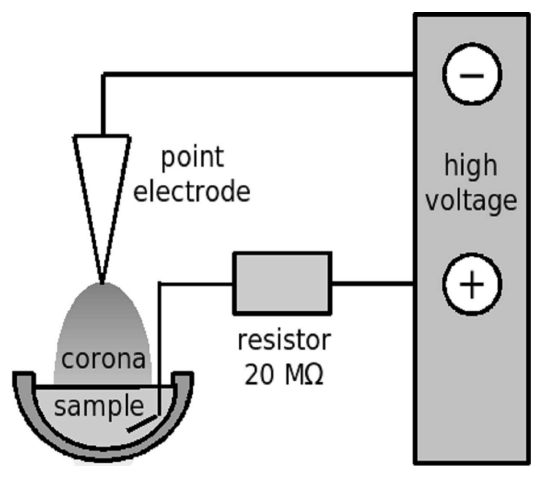

Fig. 1. Schematical arrangement of the experimental apparatus.

Due to the serial resistance in used apparatus, several burning discharges were observed. The positive point-to-plane discharge burns in the regime of positive streamer up to the current of $c a .50 \mu \mathrm{A}$ only. Varying the voltage and electrode distance, the positive discharge skips into the regime of flashing corona for currents of over $100 \mu \mathrm{A}$. This flashing corona is briefly described e.g. in [7]. In the case of negative point-to-plane discharge, it burns in 
standard pulseless glow regime, described in [8], for current up to $c a$. $200 \mu \mathrm{A}$. By shortening of the electrode distance, the current skips to value $c a .350 \mu \mathrm{A}$ and the discharge switches into a glow discharge [9]. Some electric characteristics of used discharges are described in [10]. A detailed study of these discharge types from the electrical point of view will be described after compilation in further separate study. En passant, a new form of the point-to-point discharge, called "cometary" discharge, was observed and tentatively described in [11, 12].

For the comparison of several discharge types, only positive flashing corona and both negative types were chosen. The positive streamer regime was omitted due to its low energy content and instability.

\section{Microorganisms under study}

The following microorganisms were used: a yeast $C a n-$ dida albicans and bacteria Escherichia coli, Staphylococcus epidermidis, Deinococcus radiodurans and Pseudomonas aeruginosa. Deinococcus radiodurans is the define species from Czech Collection of Microorganisms (catalogue number CCM 1700), other microorganisms are wild strains isolated in the Institute of Immunology and Microbiology, Charles University in Prague. Yeast Candida albicans is common and easy to use representative of eukaryotic microorganisms. Bacterial species were selected as a typical representatives of Gram-positive and Gram-negative ones (Staphylococcus epidermidis and Escherichia coli). The other bacteria were selected due to their distinctive attributes: Deinococcus radiodurans is an extremophile bacterium with extremely high resistance to the radiation [13]; Pseudomonas aeruginosa often causes postoperative and other nosocomial infections and is dangerous for its high resistance to many disinfection agents and antibiotics.

\section{Experiment}

Microorganisms were stored in fridge in water suspension with addition of $10 \%$ glycerol as the preservative. Before the experiment, the stock suspension was diluted to the appropriate concentration of $2 \times 10^{5} \mathrm{cfu} \mathrm{ml}^{-1}$ and $0.5 \mathrm{ml}$ of it was pipetted into the sterile wells of a dot plate, grounded and exposed to the discharge. Parameters of discharges were adjusted as follows: for the positive flashing corona to $10 \mathrm{kV}, 8 \mathrm{kV}$ or $6 \mathrm{kV}$ and $200 \mu \mathrm{A}$; for the negative pulseless glow regime to $9 \mathrm{kV}$ and $180 \mu \mathrm{A}$; for the negative glow discharge to $9 \mathrm{kV}$ and $370 \mu \mathrm{A}$. The different character of discharges determines the different parameters. The values represent a compromise between discharge stability (transition into spark or other regime) and its energy and enable the qualitative comparison of discharge types under study.

Following the exposition of water suspension, the content of each well was diluted, spread onto the surface of cultivation medium (nutrient agar for bacteria and Sabouraud agar for yeast). After the overnight cultivation at $37^{\circ} \mathrm{C}$, the numbers of survival colonies were counted. All expositions were performed under laminar flow of HEPA-filtered air to prevent the airborne contamination, the ambient conditions were controlled by an air-conditioning of the laboratory.

\section{Results and discussion}

The time dependences of the number of surviving microorganisms for each species and all discharge types are digestively shown in Figs. 2-6. The total inactivation of bacteria becomes after $60 \mathrm{~s}$ of exposition to the positive discharge and after $75 \mathrm{~s}$ of exposition to the negative one. On the other hand, exposition times of 8 or $10 \mathrm{~min}$ are necessary for the total inactivation of yeast in the case of positive or negative discharge, respectively. The best efficiency of decontamination was found for the positive flashing corona discharge at voltage $8-10 \mathrm{kV}$ and current of $200 \mu \mathrm{A}$. The negative glow-discharge is equally or more effective than the negative pulseless one. The eukaryotic yeast appeared to be more resistant to all discharge types and its inactivation required 8-10 times longer expositions.

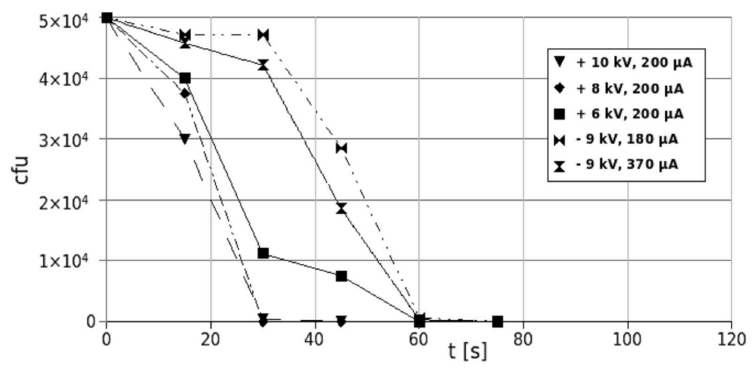

Fig. 2. The time dependences of Staphylococcus epidermidis survival under positive flashing corona, negative pulseless corona and negative glow corona discharges.

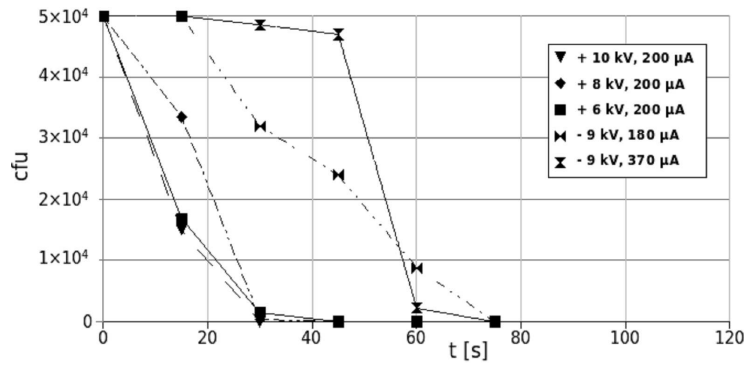

Fig. 3. As in Fig. 2, but for Escherichia coli.

The efficiency of inactivation is slightly different for various bacterial species, but no dependence on the bacterial type (Gram-negative or Gram-positive) or shape (rod or coccus) can be concluded. This result accords with our previous studies of low-temperature plasma microbicidal effects [5]. In the same work we also reported 


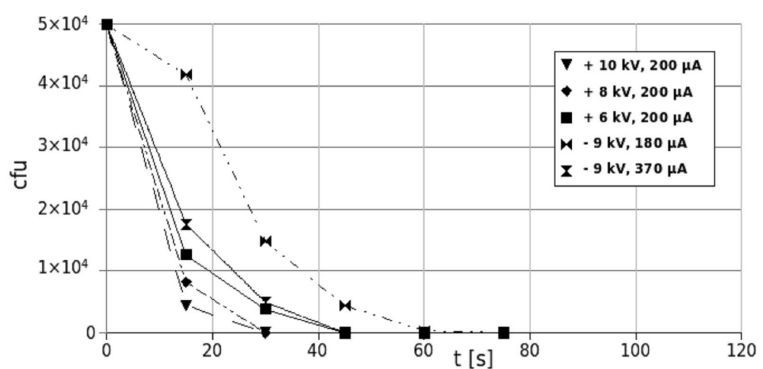

Fig. 4. As in Fig. 2, but for Deinococcus radiodurans.

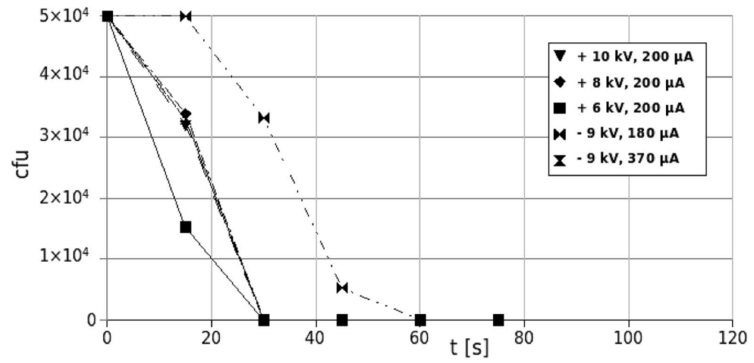

Fig. 5. As in Fig. 2, but for Pseudomonas aeruginosa.

that the yeast was the most sensitive microbe when deposited on the wet agar surface, whereas its inactivation in suspension needs several times longer exposition than bacterial suspensions. We still have no exact explanation of this phenomenon, but some hypothesis may be postulated. We suppose that:

1. The bactericidal agents are the UV radiation, ozone, free radicals and charged particles $[3,4]$.

2. UV radiation and ozone are negligible in corona discharge; free radicals and charged particles are of comparable efficiency for the inactivation of cells [14].

3. The charged particles cumulate around the cells deposited on the surface [15], but there is no cumulation of charged particles in the water suspension.

4. The yeast deposited on the agar surface displays similar or better sensitivity to the corona discharge in comparison with bacteria [5].

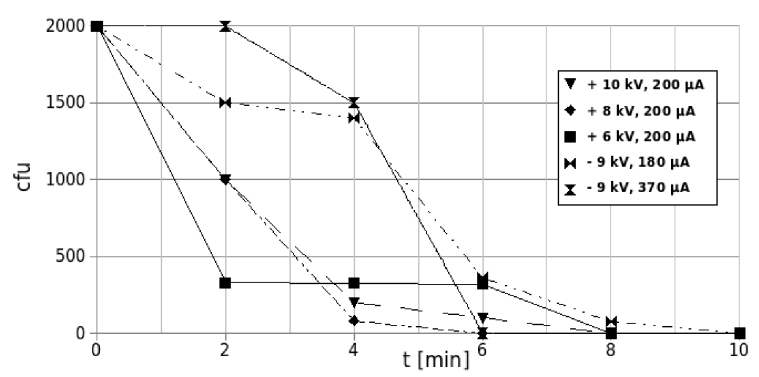

Fig. 6. As in Fig. 2, but for Candida albicans.
5. The yeast in water suspension displays lesser sensitivity to the corona discharge in comparison with bacteria.

6. The phospholipid membrane of the bacterial cell is covered and protected by the bacterial cell wall, but the yeast and other eucaryotic cells are surrounded by a phospholipid membrane only.

Consequently, we can deduce that the main reason of different sensitivity of bacteria and yeast in water suspension may be the absence of the charged particles cumulation in the water. The cumulated charged particles damage the bacterial and yeast cell equally, but the free radicals probably damage the bacterial cell wall more vigorously than the phospholipid membrane. Thus, the absence of cell wall paradoxically helps the yeast to resist the higher doses of discharge.

\section{Conclusion}

This study compares the microbicidal effect of positive flashing corona, negative pulseless corona and negative glow corona discharges in the point-to-plane arrangement in water suspension. The total inhibition of bacteria becomes after $75 \mathrm{~s}$, but the total inhibition of yeast becomes after 8-10 min of exposition. The comparatively higher resistance of $C$. albicans suggests the different mechanism of inactivation in bacteria and in eukaryotic yeasts, the hypothesis of missing cell wall for yeast cell is postulated. Further, this work documents the best efficiency of positive flashing corona at $8-10 \mathrm{kV}$. However, the range of maximal efficiency is wide and does not depend strictly on the electrical parameters, so that the precise control of these parameters will be not necessary in the future practice.

\section{Acknowledgments}

This study has been supported by grants MSM ČR 6046137306, MSM ČR 0021620806 and SVV-2010-260506 .

\section{References}

[1] J.G. Birmingham, IEEE Trans. Plasma Sci. 32, 1526 (2004).

[2] G. Fridman, A.D. Brooks, M. Balasubramanian, A. Fridman, A. Gutsol, V.N. Vasilets, H. Ayan, G. Friedman, Plasma Process. Polym. 4, 370 (2007).

[3] M. Laroussi, IEEE Trans. Plasma Sci. 28, 1409 (2002).

[4] M. Laroussi, Plasma Process. Polym. 2, 391 (2005).

[5] V. Scholtz, J. Julák, V. Kř́ha, Plasma Process. Polym. 7, 237 (2010).

[6] J. Julák, V. Křriha, V. Scholtz, Cz. J. Phys. 56, B1333 (2006).

[7] A. Fridman, L.A. Kennedy, Plasma Physics and Engineering, Routledge, USA 2004. 
[8] G.N. Trinh, J.B. Jan, IEEE Trans. PAS 87, 1207 (1968).

[9] Y.S. Akishev, M.E. Grushin, I.V. Kochetov, A.P. Napartovich, M.V. Pankin, N.I. Trushkin, Plasma Phys. Rep. 26, 172 (2000).

[10] P. Horák, J. Khun, J. Phys., Conf. Ser. 223, 012006 (2010).

[11] V. Scholtz, J. Julák, J. Phys., Conf. Ser. 223, 012005 (2010).

[12] V. Scholtz, J. Julák, IEEE Trans. Plasma Sci. 38, 1978 (2010).
[13] M.M. Cox, J.R. Battista, Nat. Rev. Microbiol. 3, 882 (2005).

[14] D.A. Mendis, M. Rosenberg, F. Azam, IEEE Trans. Plasma Sci. 28, 1304 (2000).

[15] Z. Machala, L. Chládeková, M. Pelach, J. Phys. D, Appl. Phys. 43, 222001 (2010). 